\title{
Parameters Setting and Debugging of LRDS Control Valve of Variable Pump
}

\author{
Liu Liming \\ Taiyuan Institute of China Coal Technology and Engineering Group, Taiyuan, Shanxi 030006)
}

\begin{abstract}
LRDS control valve was taken as the research object which was commonly used in coal walking machinery hydraulic system. Based on different control principles, this paper researched on their work condition and application, combined with practical engineering and maintenance experience, explored the method of parameters setting, precautions and debugging process on experiment table and machinery. The consequence provides principle for our company to specify the inspection process and maintenance regulation, and also provide methods for hydraulic system debugging and fault diagnosis.
\end{abstract}

\section{INTRODUCTION}

Axial plunger variable pump with swash plate structure has the advantages of large power density, wide stepless speed regulation range, various variable control methods, easy to achieve overload protection and self-lubrication, which is used for hydrostatic transmission in open circuit hydraulic systems, which is designed for machines marching.It can adapt to the complicated working conditions of the whole machine equipment through continuous changes in displacement. Since the variable displacement pump has only one controlled object of displacement, when different control methods are used, the variable displacement pump can have different output characteristics [1-2].So it is meaningful to the machine equipment hydraulic system debugging, rapid fault diagnosis, component selection and maintenance, overhaul and so on ;moreover it can aslo guide the design of hydraulic system, improvement of hydraulic components.

\section{BRIEFN INTRODUCTION OF LRDS CONTROL FUNCTION}

At present, although there are many manufacturers of variable pumps, in terms of control functions, valvecontrolled pumps are used as the main method to form diversified composite control functions such as LE, LRC, DRS, and LRDS.LRDS is a commonly used control function of coal machine equipment hydraulic system. Its working principle is as below: LR (constant power) belongs to process control, D (constant pressure control) and $\mathrm{S}$ (load sensitive) belong to state control. In the figure, LR represents constant power control valve, D represents constant pressure control valve, and $\mathrm{S}$ represents load sensitive valve.) Indicates a throttle valve or multi-way valve, T1 and T2 are drain ports, $\mathrm{R}$ is a bleed port, $\mathrm{M}$ and $\mathrm{M} 1$ are pressure measuring ports, $\mathrm{A}$ is an oil inlet, and $\mathrm{S}$ is an oil inlet, Vgmax / Vgmin represent the maximum / minimum displacement, respectively.

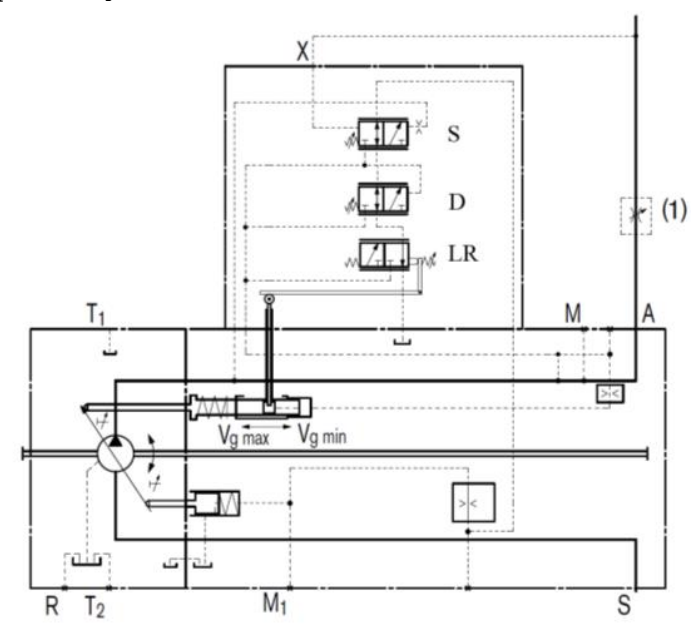

Figure1. LRDS Control Function Schematic

The LRDS control characteristic curve is shown in Figure 2.The curve and the area below it are the effective working area of the plunger pump, $\mathrm{p}_{\mathrm{D}}$ is the set value of constant pressure control valve, $\mathrm{pLR}$ is the set value of constant power starting pressure at maximum displacement. The effective working area of the piston pump is composed of three parts:1)The dashed part, that is, the shaded part below the hyperbola and horizontal straight line, only the load-sensitive valve is in the working state;2) Along the horizontal straight line, only the constant pressure control valve is in the working state;3) Along the hyperbola, only the constant pressure control valve is in working condition. Three different control functions are arranged in parallel in the entire system. Under any working pressure and output flow state (pi, Qi), only one valve is in working state. The working pressure and output flow determine the working 
sequence and logical control of the three relationships. The different working areas are described in detail as below:

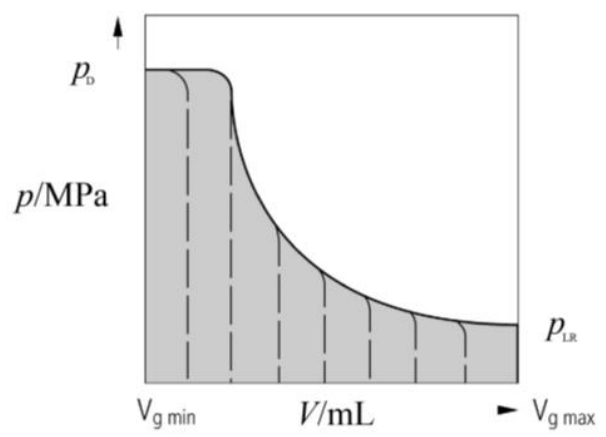

FIG 2. LRDS

\subsection{Dashed line, $\mathrm{S}$ control function}

In this area, that is, the shaded part below the horizontal straight line and the hyperbola, the working pressure does not reach the constant pressure control value, the output power does not reach the constant power hyperbola value, and only the load-sensitive valve is in the working state. The load-sensing valve is essentially a flow control valve. The output flow of the variable pump is only affected by the external throttle valve (or loadsensing multi-way valve) installed between the pump and the actuator, as shown in Figure 1 (1). The working principle is shown in the $\mathrm{S}$ valve in Figure 1. The loadsensing valve compares the pressure upstream and downstream of the orifice and keeps the throttle pressure drop $\Delta \mathrm{p}$ constant, so that the output flow of the variable pump remains constant. If the throttle valve opening is reduced, the pressure difference $\Delta \mathrm{p}$ at both ends of the throttle valve increases, the swash plate swings in the direction of Vgmin, and the output flow decreases. As $p$ decreases, the swash plate swings toward Vgmax, and the output flow increases. In the whole control process, the displacement is only related to the size of the throttle opening, and has nothing to do with the load pressure [3]. The load-sensing valve balance equation is:

$$
\Delta p=p_{0}-p_{\mathrm{L}}=F_{\mathrm{S}} A_{\mathrm{S}}
$$

In the equation:

$F_{\text {S-Load-sensing valve adjustable spring force; }}$

P0-Output pressure of plunger pump;

pL-Load pressure;

AS-Effective area of load feedback pressure;

$\triangle p$-The pressure difference between the two ends of the spool, that is, the pressure difference between the upstream and downstream of the external throttle valve.

\subsection{Hyperbolic part, LR control function}

Along the hyperbola, only the constant power valve is in operation. The constant power control function controls the working pressure and output flow through a variable mechanism, so that the set driving power is not exceeded at a constant speed. The constant power control function of the axial piston variable pump has evolved from the double spring displacement feedback mechanism with two straight lines that are approximately hyperbolic in the past to the current lever torque balance control mechanism that is approximately completely hyperbolic. The precise control using hyperbolic characteristics can achieve the available power The best use of the improved the output characteristics of the constant power control function to a certain extent, but the degree of approximation of the hyperbola and the accuracy and stability of the control process depend entirely on the constant power control mechanism and the variable displacement mechanism of the plunger pump. Interaction [4-5]. The working principle is shown in the LR valve in Figure 1. The constant power valve spool determines the working position according to the torque balance relationship between working pressure and spring force. If the working pressure exceeds the set constant power starting control pressure, the control valve spool moves to the right, the working pressure acts on both the variable plunger and the variable piston at the same time, and the swash plate swings in the direction of Vgmin under the force of the lever, so that the exhaust The amount decreases in the same proportion that the working pressure increases without exceeding the predetermined driving power. As shown in formula (2), The constant power prime mover and variable pump achieve the best match to ensure that the prime mover works under the best working conditions, reduce energy consumption, and play an overload protection role. This function is essentially a constant product of working pressure and displacement.

$$
P V_{g} n \times 10^{-3}=C
$$

In the formula:

p-working pressure, $\mathrm{MPa}$;

Vg-displacement, ml;

$\mathrm{C}$-power setting value, $\mathrm{kW}$;

$\mathrm{n}$-speed, $\mathrm{r} / \mathrm{min}$.

\section{$2.3 \mathrm{D}$ control function, while $\mathrm{pD}=p$}

Along this line, only the constant pressure valve is in operation.Constant pressure control keeps the pressure in the hydraulic system constant within its control range and has nothing to do with the changing pump output flow. The variable pump only supplies the flow required by the actuator.The working principle is shown as D valve in Figure 1. If the working pressure exceeds the set pressure, the pump will swing back to a smaller angle automatically.In non-working state, under the action of the constant pressure valve control spring, the variable pump swash plate swings back to the starting position (Vgmax).

The constant pressure pump system replaces the overflow valve control system. In the constant pressure variable pump control system, the excess flow is not discharged from the overflow valve. The change in displacement is used to meet the system's flow demand, so there is no overflow loss and savings It reduces energy consumption, reduces system heating, prolongs the service life of hydraulic oil and components, and reduces the operating cost of hydraulic systems [6-7]. 


\section{COMMISSIONING OF LRDS VALVE}

Considering the priority and control logic relationship of different control functions of LRDS valve, first debug S valve, second LR valve, and finally $\mathrm{D}$ valve. According to different occasions of the valve on the test bench or the whole equipment, the specific debugging method is shown below.

\section{1 $\mathrm{S}$ Commissioning of the valve}

If the component under test is in a test system equipped with a closed-center load-sensitive multi-way valve, or when it is installed and debugged on the whole machine, the multi-way valve is in the neutral position, and the output pressure after the pump starts is the set pressure of the load-sensitive valve The setting range is between 1.4 $\mathrm{MPa}$ and $2.5 \mathrm{MPa}$, and the standard value is usually $1.8 \mathrm{MPa}$ [8].Turn the load-sensing valve adjustment screw clockwise to increase the pressure. Turn the loadsensing valve adjustment screw counterclockwise to reduce the pressure.From a safety perspective, to ensure that the initial pressure will not be too high, the loadsensing valve adjustment screw should be loosened counterclockwise before starting the pump. After starting the pump, gradually turn the adjustment screw clockwise until the pressure reaches the set value.Due to the pressure loss along the process, the pressure difference between the two ends of the multi-way valve orifice during normal operation of the load-sensitive system is slightly smaller than the pressure difference at the neutral position of the multi-way valve. Therefore, this debugging method makes the standby pressure of the pump slightly higher than normal The pressure difference between the two ends of the orifice of the multi-way valve.

If the component under test is tested on a common throttling test bench, debug according to the following procedure:

1) Connect the pump inlet, outlet, and drain ports of the pump correctly, connect the load sensing port of the control valve to the fuel tank, and loosen the load sensing valve adjustment screw counterclockwise until the pump's working pressure and output flow are almost zero. According to the load-sensing valve balance equation (2), because the load feedback pressure is zero, the output pressure of the pump is equal to the set pressure of the load-sensing valve, that is, the pressure difference $\Delta \mathrm{p}$ across the orifice.

2) Turn the pointer into the load-sensing valve adjustment screw until the pump has a flow output, and then load the pump. If the output flow becomes zero before the pressure reaches the load-sensing valve setting value, continue to turn the pointer into the adjustment screw (fine adjustment, each time about Halfturn or 1 / 4-turn) Continue to load the pump until there is a flow output, and repeat the operation until the pump's working pressure reaches the set value and the output flow is zero.

\subsection{Commissioning of LR valve}

Constant power control works only when the working pressure is within the set range, so the parameter adjustment of the LR valve is the setting of the initial control pressure, and the effective set value is between $5 \mathrm{MPa}$ and $32 \mathrm{MPa}$, as shown in Figure 3. The LR valve needs to be debugged in a test system that can simultaneously detect the working pressure and output flow. The whole equipment is usually only pressure display, so it can be roughly estimated by the movement speed of the actuator under different pressures or the torque output by the motor in the electronic control system. The test procedure of the valve on the test bench is as follows:

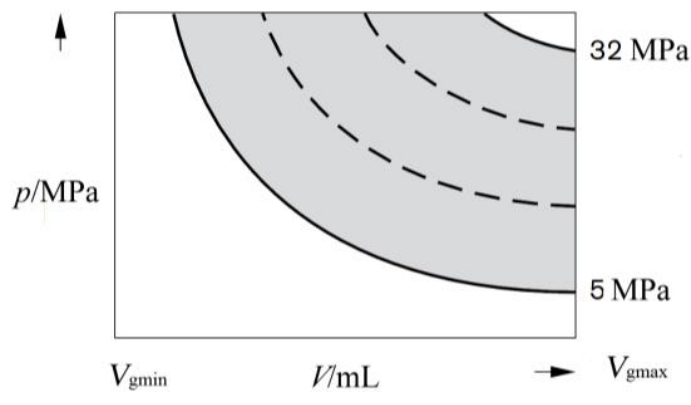

Fig3. Constant power control curve

(1) Connect the pump inlet and outlet and drain ports properly. Connect the load sensing port of the control valve with the pump pressure measuring port $M$ to shield the load sensing function and lock the constant pressure control valve adjusting screw clockwise to avoid interference between the load sensing and constant pressure control functions during the LR valve debugging process.

(2) Start the pump and gradually load it to the set pressure value after the operation is stable. If the output flow detected by the flow meter at this time is less than the maximum output flow of the pump (indicating that the initial control pressure is lower than the target value), turn it into the constant power valve clockwise Adjust the screw; if the output flow is equal to the maximum output flow of the pump at this time (indicating that the initial control pressure is higher than the target value), turn the constant power valve adjustment screw counterclockwise until it starts to load from the set value, and the output flow follows the working pressure Increase until the corresponding decrease.

(3) During the loading process, in the constant power action range, the corresponding output flow under different pressures is recorded, and the output power is calculated at the same time to ensure the correctness of parameter debugging. Considering the existence of the principle error in the constant power control process and the decrease in volumetric efficiency as the working pressure increases, the power value decreases slightly as the working pressure increases. Therefore, according to the actual operating conditions, The power at the initial control pressure or rated working pressure is set to a standard value. 


\subsection{Commissioning of $D$ valve}

The constant pressure control valve mainly protects the system from excessive pressure. The effective parameter setting range is between $5 \mathrm{MPa}$ and $35 \mathrm{MPa}$.If debugging on the whole machine, from a safety perspective, in order to prevent the system pressure from being too high, you can first loosen the adjusting screw of the constant pressure control valve, and then start the oil pump to limit the action of the actuator with external conditions (for example, push the cylinder to the end) The pressure displayed by the system pressure gauge is the working pressure of the constant pressure control valve, and gradually turn the adjustment screw of the constant pressure control valve clockwise until the pressure value reaches the requirement.

If debugging on the test bench, follow these steps:

(1) Connect the inlet and outlet and drain ports of the pump correctly. Connect the load sensing port of the control valve with the pump pressure measuring port $\mathrm{M}$ to shield the load sensing function and avoid the interference of the load sensing on the D valve during the debugging process. Before starting the motor, lock the constant pressure control valve adjustment screw clockwise.

(2) Start the drive motor, and after the plunger pump runs stably, gradually load the working pressure to the set pressure value $\mathrm{p}$ (assuming $\mathrm{p}=25 \mathrm{MPa}$ ). At this time, because the adjusting screw of the constant pressure control valve has been locked, the valve is not working State, gradually turn out the constant pressure control valve adjusting screw counterclockwise. When the pump output flow rate is zero, the valve is in working state. The output pressure of the pump is equal to the setting pressure of the constant pressure control valve spring $25 \mathrm{MPa}$.

(3) After the plunger pump is depressurized and reloaded, when the pressure reaches the above set value, the output flow of the pump quickly drops to approximately zero. Generally, the set pressure of the constant pressure control valve is higher than the constant power starting control pressure. When the output pressure of the pump is close to the constant pressure valve set value, the constant power valve is in the working stage, so the output flow of the pump will be reduced accordingly, but it will not be reduced to Zero (don't think that the constant pressure control valve starts to work), while taking into account that the response of the hydraulic test system has a certain lag, so adjust the screw in / out of the adjustment screw, and stop the pressure gauge and flow every half or $1 / 4$ turn table.

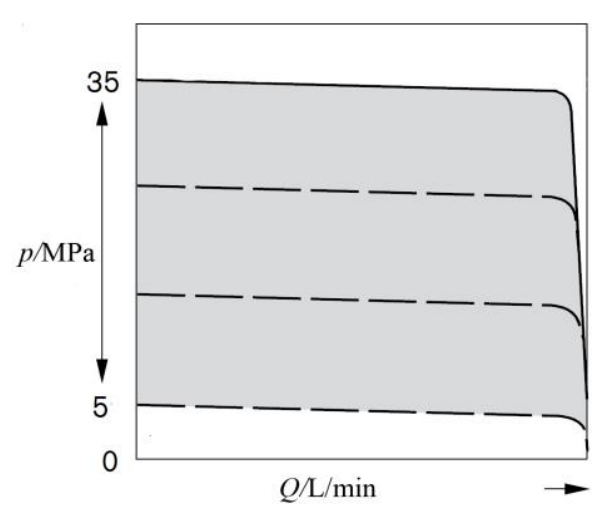

FIG4.Constant voltage control curve

\section{Practical application}

Taking a certain type of roadheader as an example, the practical application of the LRDS control function and the solution of common faults are introduced.Figure 5 is a hydraulic system diagram of a certain type of roadheader. It belongs to a typical LRDS control circuit. The entire system is powered by a double pump. The first plunger pump drives a transport, loading motor, and bolting machine through a quadruple multiplex valve. And spray motor; the second plunger pump drives seven cylinders, including various types of cylinders (including crawler tension cylinders, cutting head rotation, lifting, telescopic cylinders, shovel cylinders, rear support cylinders).The working pressure of the plunger pump is determined by the maximum load pressure, and the output flow is controlled by the size of the multi-way valve opening.

1)Start the oil pump motor. When the multi-way valve is in the neutral position, the system is in a lowpressure standby state. The output flow of the plunger pump is almost zero, which only meets the necessary internal leakage and lubrication. The working pressure is about 1.8MPa. The setting pressure of the load-sensing valve directly affects the working speed of the actuator, such as the speed of walking. If the pressure is small, the speed is slow, and the pressure is too fast. Therefore, in the low-pressure standby state, you can observe the pressure gauge and adjust the load-sensitive valve according to the above method to make the working pressure reach the standard set value.

2)Operate the multi-way valve and limit the displacement of the actuator. As shown in the oil cylinder in Figure 5, the output flow of the plunger pump is almost zero, which only meets the internal leakage and lubrication under high pressure; the output pressure of the plunger pump is the working pressure of the constant pressure valve About $25 \mathrm{MPa}$. The constant pressure valve mainly plays a role of high pressure protection to prevent the system pressure from being too high. In this state, you can observe the pressure gauge and adjust the constant pressure valve according to the above method to make the working pressure reach the standard set value.

3)Because the whole machine is usually not equipped with a flow meter, as mentioned earlier, the power setting is roughly estimated through the parameters such 
as the motor torque or current displayed by the electric control box, or the power is estimated through the working speed of the actuator under high pressure based on experience. Size, this function is best adjusted on the test bench. The constant power valve is mainly used for overload protection, limiting the maximum power of the system and preventing the oil pump motor from being overloaded.

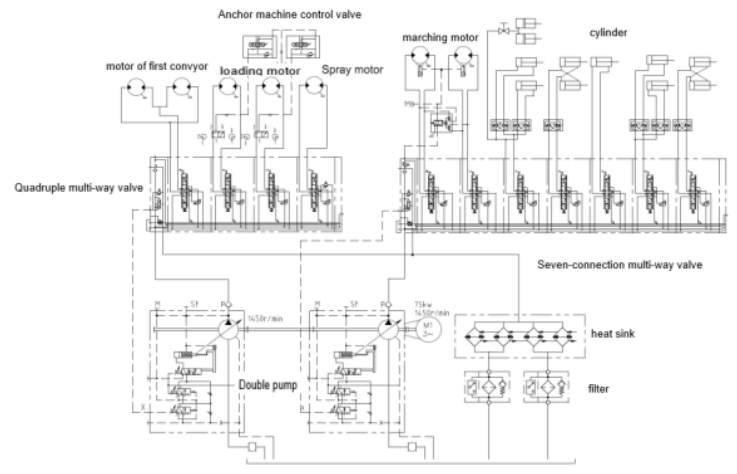

FIG 5: Roadheader hydraulic system diagram

\section{5.conclusion}

On the basis of analyzing the principle of LRDS control function, this paper, from the perspective of practical engineering application, combined with its own working experience, for different occasions, discusses the setting method of LRDS control function parameters, and precautions in the debugging process. The determination of the setting method and the debugging process has a certain guiding effect on the factory inspection, overall system debugging and fault diagnosis.

\section{References}

1. LIU Zhao, ZHANG Shan-shan. Variable pump control 's method and its application. [J]. CHINESE JOUNRAL OF CONSTRUCTION MACHINERY, 2004, 2(3), 304-307.

2. TANG Zhao-jian; LUO Jian-guo; CHENG Hao.The Research of Reliability Test Platform for Plunger Pump in Profiling Buoy[J]. Hydraulic and pneumatic, 2016. 04.

3. YIN Jie; ZHANG Jian-min; ZHANG Yuan-shen. The Dynamic Characteristic Analysis of Load Sensing Axial Piston Pump Based on the AMESim. [J].Hydraulic and pneumatic, 2014, 07.

4. XUE Yafeng.Study of the Constant Power Control Principle Error of Variable Pump with Inclined Piston[J]. MACHINE TOOL \& HYDRAULICS, 2016, 44(2), 89-92.

5. DENG Bo-wen, ZHU Jian-gong. The Research of Constant Power Control Program for Piston Variable Pump.[J]Hydraulic and pneumatic, 2009(8), 41-42.

6. Mo Bo, Lei Ming, Cao Fan.A Principle to Adjust the Volume Control of Constant Power and
Constant Pressure Pump. [J]Hydraulic and pneumatic, 2002, 6, 5-6.

7. LIU Qing-xiu.Simulation Analysis on Constant Pressure Variable Displacement Pump Based on AMESim [J].Coal Mine Machinery, 2011, 32(6), 129-131. 